\title{
Die Acidität des Magensaftes von Scyllium stellare.
}

\section{Von}

\author{
M. A. v. Herwerden und W. E. Ringer.
}

Mit einer Kurvenzeichnung im Text.

(Aus dem physiologischen Laboratorium der Universität Utrecht).

(Der Redaktion zugegangen am 18. September 1911.)

Vor einiger Zeit haben wir $\left.{ }^{1}\right)$ Versuche über die Säureabscheidung in der Magenschleimhaut von Selachiern angestellt. Dabei wurde nach der Methode Sjöqvists die Art der Säure $z u$ erforschen gesucht. Aus den Versuchen wurde der Schluß gezogen, daß wenigstens die Hauptmenge der Säure Salzsäure sein müsse. Daneben konnte (durch Destillation) eine kleine Menge Ameisensäure aufgefunden werden. Es wurde aber schon damals der Schluß gezogen, daß «die Methode Sjöqvists für quantitative Bestimmungen bei der Untersuchung des Magensaftes von Seetieren in der Zukunft nicht genügen würde und durch eine andere ersetzt werden müsse».2)

v. Herwerden hat schon damals den Einfluß von Erdalkalisalzen erkannt und gefunden, daß im Gegensatz zu den ursprünglichen Sjöqvistschen Angaben diese zu bedeutenden Fehlern Anlaß geben. ${ }^{3}$ )

Von Weinland ${ }^{4}$ ) ist neuerdings die Unzulässigkeit der Sjöqvistschen Methode nochmals betont worden. Dieser Forscher findet, daß Calciumchlorid ohne jeglichen Säurezusatz

1) M. A. v. Herwerden, Diese Zeitschrift, Bd. 56, S. 453 (1908).

2) S. 464 .

3) Der Vorwurf Weinlands (Zeitschrift f. Biologie, Bd. 55, S. 58 (1910), daß v. Herwerden keine Versuche darüber angestellt hat, ist also unrichtig, man siehe Tabelle II, S. 461, der zitierten Abhandlung v. Herwerdens.

4) Zeitschrift f. Biologie, Bd. 55̃, S. 58 (1910). 
nach der Sjöqvistschen Methode Säure vortäuscht. Durch Zusatz organischer Säure wird dieser «Säuregehalt» erhöht. So wurde mit $25,5 \mathrm{ccm} n / 10-\mathrm{CaCl}_{2} 35,5 \mathrm{ccm} n / 10-\mathrm{HCl}$ nach $\mathrm{Sjöqvist}$

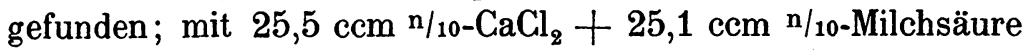
aber $36,5 \mathrm{ccm} \mathbf{n} / 10-\mathrm{HCl}$. Mit dieser beträchtlichen Menge Milchsäure wird also doch nur wenig «Salzsäure» mehr gefunden. was der kleinen Dissoziationskonstante der Milchsäure entspricht. Weil aber v. Herwerden viel beträchtlichere Unterschiede zwischen dem Salzsäurewert, welchen nach der Sjöqvistschen Methode die Salze des Meerwassers vortäuschen, und demjenigen, welchen das in den Magen eingeführte und später ausgeheherte Seewasser erweist, gefunden hat, kann die Acidität des Magensaftes nicht von einer schwachen organischen Säure herrühren.

Wir waren jetzt in der Lage, die Versuche wieder aufzunehmen. Nach einer gleich zu beschreibenden Methode haben wir den Magensaft von Scyllium untersucht. Der Saft wurde von uns in der zoologischen Station zu Neapel in folgender Weise gesammelt. Die Tiere bekamen Fibrin (in späteren Versuchen in Glycerin bewahrt, vor dem Gebrauch aber gründlich ausgewaschen. Es ist bemerkenswert, daß besonders mit diesem Fibrin eine überaus reichliche Saftabscheidung erfolgte) oder auch in einigen Versuchen Sardinen. Nach 24 Stunden (bei den Sardinenversuchen nach $3 \times 24$ Stunden) wurde der Magen mit der Sonde entleert, der Mageninhalt filtriert, Thymol zugesetzt, nach dem hiesigen physiologischen Laboratorium verschickt und sofort untersucht.

Bei der Untersuchung zeigte sich, daß der Magensaft stark sauer reagierte. Wir haben die H-Ionenkonzentration elektrometrisch gemessen und dieselbe oft in der Nähe von $0,02 n$ $\left.\left(p_{H}=1,69\right)^{1}\right)$ gefunden. Es zeigte sich auch, daß, wie zu erwarten war, der Saft einen großen Gehalt an Seewasser hatte; daneben waren natürlich die Digestionsprodukte des Fibrins

1) Über die Bedeutung von $p_{H}$ siehe: Sörensen, Etudes enzymatiques, Compt. rend. d. Trav. d. Laboratoire de Carlsberg, 8me volume, 1re livraison, 1909. 
oder der Sardinen zugegen. Es lag also ein sehr kompliziertes Gemisch vor.

Die Frage war, welche Säure oder Säuren hat die Magenwand ursprünglich abgeschieden; ob im vorliegenden Saft «freie» Salzsäure oder eine andere «freie» Säure anwesend war, war hier unwesentlich. Nachdem wir einmal gefunden hatten, daß der Saft sauer reagierte und zwar ziemlich stark, konnte man natürlich sowohl von freier Salzsäure wie von Schwefelsäure reden, weil beide Säuren im Meerwasser als Salze vorkommen. Eine "positive Reaktion» auf Salzsäure sagt also nicht viel aus. Nur konnten wir aus der ziemlich großen $\mathrm{C}_{\mathrm{H}}$ den Schluß ziehen, daß, welche Säure die Magenwand auch abgeschieden hat, sie auf jeden Fall keine schwache sein kann. Schon deswegen können wir vermuten, daß es nicht eine organische Säure ist. Die am meisten auf der Hand liegenden Säuren sind wohl die Ameisensäure, $\mathrm{K}=0,000214$, und Milchsäure, $\mathrm{K}=0,000138$, die also beide noch ganz schwach sind. Auch die übrigen hier in Betracht kommenden organischen Säuren, wie Essigsäure $\mathrm{K}=0,000018$, Propionsäure 0,0000134, Buttersäure 0,0000149, $\beta$-Oxypropıonsäure 0,0000311 oder Glycerinsäure $K=0,000228$, können ihrer Schwäche wegen doch wohl kaum die Acidität des Haifischmagensaftes hervorbringen.

Damit z. B. die Ameisensäure oder die Milchsäure im Magensafte eine $\mathrm{C}_{\mathrm{H}}=0,02$ hervorbringe, müßte ihre Konzentration sehr beträchtlich sein. Wir wissen, daß ein Teil der Säure von den Eiweißabbauprodukten "gebunden» wird. Nehmen wir an, daß ohne diese Abbauprodukte die $\mathrm{C}_{\mathrm{H}}=0,04$ wäre, so gehen wir dabei sicher nicht zu weit. Damit aber die Ameisensäure eine $\mathrm{C}_{\mathrm{H}}=0,04$ hervorruft, muß ihre Konzentration 7,52 $\mathrm{n}$ sein. ${ }^{1)}$ Für die Milchsäure ergibt sich die Zahl 11,63 n. Sollte also die Acidität des Magensaftes von einer organischen Säure herrühren, so müßte dieselbe eine sehr viel stärkere als die

1) Sei $\mathrm{c}$ die Konzentration, $\alpha$ der Dissoziationsgrad, $\mathrm{C}_{\mathrm{H}}=0,04$, so ist:

$\mathrm{K}(1-\alpha)=\mathrm{c} \alpha^{2} ; \mathrm{c} \alpha=0,04 ; \mathrm{K}=0,000214$ oder $\alpha=0,00532$ und $\mathrm{c}=7,52$

für die Milchsäure, $\mathrm{K}=0,000138$, ist $\alpha=0,00344$ urd $\mathrm{c}=11,63$. 
zwei genannten sein; die Gegenwart einer so starken organischen Säure ist aber an sich schon unwahrscheinlich. Damit ist aber nicht gesagt, daß im Magensaft gar keine organische Säure vorkommt. Eine solche könnte durch die chemischen Prozesse im Magen gebildet werden, sie könnte auch in sehr geringen Mengen von der Magenwand sezerniert oder auch wohl als Salz abgeschieden werden, woraus dann aber die stärkere Säure des Saftes die schwächere in Freiheit setzt.

Bei unseren Untersuchungen gingen wir nun von folgenden Überlegungen aus. Der Magensaft enthält anorganische oder organische Säuren oder beide. Wird der Saft neutralisiert, z. B. mit $\mathrm{NaOH}$, so binden diese Säuren eine ihnen äquivalente Basenmenge. Beim neutralen Punkte werden die übrigen Bestandteile, insbesondere die amphoteren Eiweißabbauprodukte keine merkliche Basen- oder Säuremenge für sich in Anspruch nehmen. Die zum Neutralisieren verwendete Basenmenge hat nur die im Magensaft vorhandenen Verbindungen von bestimmt saurem Charakter abgesättigt. Man findet auf diese Weise also die gesamte Säuremenge.

Wird die neutrale Flüssigkeit zur Trockenheit eingeengt und eingeäschert, so entweicht dabei ein Teil des Chlors; infolge der Gegenwart von Meerwasser sind nämlich beträchtliche Mengen von $\mathrm{Ca}$ und $\mathrm{Mg}$ zugegen. Daneben entsteht bei dem Verbrennen der Digestionsprodukte immer etwas Schwefelsäure durch Verbrennung des Schwefels aus den Eiweißprodukten. Die so gebildete Schwefelsäuremenge zeigte sich bei der Untersuchung aber als sehr gering und war also zu vernachlässigen. Dann aber entweicht neben Salzsäure auch Schwefelsäure aus dem Meerwasser und der Schwefelsäureverlust kann unter Umständen beträchtlich sein, wie die Versuche zeigten.

Wird die Asche in verdünnter Säure gelöst, so wird von dieser ein Teil gebunden und zwar soviel, als den verflüchteten Säuremengen äquivalent ist ${ }^{1}$ ) (bei Vernachlässigung der aus dem Eiweißschwefel gebildeten Schwefelsäurespur). Waren aber

1) Die Sulfate werden bei der Verbrennung der Asche zum Teil reduziert, dann zum Teil wieder zu Sulfat oxydiert oder auch wohl zu Oxyd, der letzte Teil gibt zu Säurebindung Anlaß. 
organische Säuren vorhanden, so wird auch noch eine damit äquivalente Säuremenge gebunden, weil die organischen Salze beim Verbrennen in Carbonate übergeführt worden sind.

Bestimmt man also die verflüchtigten Salzsäure- und Schwefelsäuremengen und das Säurebindungsvermögen der Asche, so kann man aus dem Vergleich der erhaltenen Werte auf das eventuelle Vorhandensein organischer Säuren schließen.

Die beschriebenen Voraussetzungen haben wir dann durch Versuche auf ihre Stichhaltigkeit geprüft.

1. Zuerst haben wir uns die Frage vorgelegt, wie verhält sich der Eiweißschwefel beim Einengen und Verbrennen des neutralisierten Magensaftes?

2. Stimmt die Alkalität der Asche bei Abwesenheit organischer Säuren mit dem Salzsäure- und Schwefelsäureverlust überein?

3. Wird zugesetzte organische Säure in der beschriebenen Weise wiedergefunden?

Wir stellten uns künstlichen Magensaft her aus vielfach ausgewaschenem Fibrin und Pepsinsalzsäure. Das Fibrin wurde 24 Stunden bei $37^{\circ}$ digeriert. Die so erhaltene Lösung gab nach Filtration und Verdünnung mit $\mathrm{BaCl}_{2}$ keine Schwefelsäurereaktion.

Versuch I. $10 \mathrm{ccm}$ der Lösung wurden mit $\mathrm{NaOH}(0,1141 \mathrm{n})$ neutralisiert. Verbraucht wurden $4,19 \mathrm{ccm} \mathrm{NaOH}$. Die Titration haben wir nach einigen Vorversuchen mit verschiedenen Indikatoren, zum Schluß mit empfindlichem Lackmuspapier vorgenommen. Äußerst schmale Streifchen wurden dazu benutzt. Nach einiger Übung gelingt es, sich des neutralen Punktes genügend zu nähern, die Kontrollversuche wiesen nur kleine Differenzen auf. ${ }^{1}$ )

Die Acidität der Lösung war also 0,0478 n. Gefunden wurde weiter $0,0304 \mathrm{~g}$ Asche. Das zum Neutralisieren zugefügte $\mathrm{NaOH}$ hat $0,0279 \mathrm{~g}$ $\mathrm{NaCl}$ gebildet. Die Asche wurde gelöst und die Lösung war alkalisch. Die Titrationsalkalität war, als die Lösung auf das Anfangsvolumen gebracht wurde $(10 \mathrm{ccm}), 0,0046 \mathrm{n}$. In dieser Lösung wurde eine Schwefelsäurebestimmung vorgenommen, dazu wurde sie etwas verdünnt und mit

1) Wegen des Vorhandenseins amphoterer Spaltungsprodukte des Fibrins konnte selbstverständlich nur ein Indikator verwendet werden, der den neutralen Punkt möglichst nahe angibt. So war Phenolphthalein gar nicht brauchbar. 
Salzsäure angesäuert. In der Siedehitze wurde die Schwefelsäure mit $\mathrm{BaCl}_{2}$ in geringem Überschuß präzipitiert, sodann noch eine Zeitlang auf dem Wasserbade erhitzt und 24 Stunden bei Zimmertemperatur stehen gelassen. 1) Filtriert wurde durch Dreverhoffs Barytfilter Nr. 400 . Gefunden wurde nur $0,0018 \mathrm{~g} \mathrm{BaSO}_{4}$.

Aus diesem Versuch sehen wir, daß der Schwefel des Fibrins nur für einen sehr kleinen Teil zu $\mathrm{SO}_{3}$ oxydiert wird. Aus dem folgenden Versuch ergibt sich nämlich, daß der Gesamtschwefel des Fibrins $0,0277 \mathrm{~g} \mathrm{BaSO}_{4}$ hätte bilden können. Also würde etwa 6\% des Schwefels oxydiert sein. Die Schwefelsäurebestimmung ist aber bei einem so geringen Gehalt selbstverständlich nicht genau.

Weiter sehen wir, daß die Asche etwas alkalisch ist. Beim Erhitzen einer geringen Menge Chlornatriums in Gegenwart eines großen Überschusses der Eiweißabbauprodukte bildet sich also doch eine kleine Menge Natriumcarbonat.

Versuch II. $10 \mathrm{ccm}$ der Lösung vermischt mit $20 \mathrm{ccm} \mathrm{NaCl} \mathrm{1/10} \mathrm{n}$ (=0,1169 $\mathrm{mg} \mathrm{NaCl}$ ). Titrationsacidität 0,0456 (Differenz mit der vorigen Bestimmung also 0,0022). Bei der Titration gebildet $0,0267 \mathrm{~g} \mathrm{NaCl}$. Trockenrückstand $0,4896 \mathrm{~g}$. Asche $0,1475 \mathrm{~g}$. Glühverlust $0,3421 \mathrm{~g}$. Alkalität der Asche 0,0051 n..$^{*}$ In der Lösung gefunden 0,0028 $\mathrm{g} \mathrm{BaSO}_{4}$.

Aus diesem Versuch ergibt sich, daß auch bei größerem Gehalt der Asche an Salz $(0,1169 \mathrm{~g}+0,0267 \mathrm{~g}=0,1436 \mathrm{~g} \mathrm{NaCl})$ die Alkalität der Asche von ganz derselben Größenordnung bleibt. Auch hier ist nur ein ganz kleiner Teil des Schwefels oxydiert.

Versuch III. $10 \mathrm{ccm}$ Lösung $+20 \mathrm{ccm} \mathrm{NaCl} 0,1 \mathrm{n}$. Titrationsacidität 0,0478 . Trockenrückstand $0,4913 \mathrm{~g}$. Asche $0,1477 \mathrm{~g}$. Alkalität der Asche 0,0062. Die Asche war hier statt mit Salzsäure mit Schwefelsäure in Lösung gebracht und mit $\mathrm{NaOH}$ zurücktitriert. ${ }^{3}$ ) Chlorgehalt der Asche, nach Abzug des Chlors der $20 \mathrm{ccm} \mathrm{NaCl} \mathrm{0,1} \mathrm{n,} \mathrm{0,0437} \mathrm{n.} \mathrm{Ge-}$ samtchlorgehalt, durch Verbrennen unter Zugabe von chlorfreiem $\mathrm{KNO}_{3}$

1) $\mathrm{BaSO}_{4}$ ist in chloridhaltiger Lösung bei höherer Temperatur merklich löslich.

2) Also die Alkalität der Lösung der Asche, wenn diese Lösung auf das Anfangsvolumen gebracht wird (10 ccm).

s) Zur Bestimmung der Aschenalkalität wurde die Asche mit Wasser und einem Überschuß titrierter Säure, $\mathrm{HCl}$ oder $\mathrm{H}_{2} \mathrm{SO}_{4}$, eine Zeitlang auf dem Wasserbade erhitzt. Dabei wurde entweder eine ganz klare Lösung oder eine Lösung mit Spuren Kohlenstoffteilchen erhalten. Nach dem Erhitzen wurde die überschüssige Säure zurücktitriert. 
und $\mathrm{NaCO}_{3}$ bestimmt, Mittelwert aus zwei Versuchen 0,054 $\mathrm{n}$. Chlorverlust 0,0103. Dieser Chlorverlust muß der Aschenalkalität + der aus dem Fibrinschwefel gebildeten Schwefelsäuremenge gleich sein, denn die gebildete Schwefelsäuremenge hat eine äquivalente Chlormenge ausgetrieben. Aus dem gefundenen $\mathrm{BaSO}_{4}(0,0028 \mathrm{~g}$ aus $10 \mathrm{ccm})$ ergibt sich eine Schwefelsäurenormalität von $0,00240 \mathrm{n}$. Also Aschenalkalität + gebildete Schwefelsäure $=0,0062+0,0024=0,0086 \mathrm{n}$. Dies stimmt bis auf sehr wenig mit dem Chlorverlust 0,0103. Die Differenz dürfte wohl Analysenfehlern zuzuschreiben sein.

Also, der Eiweißschwefel wird bei unserer Methode zu einem sehr geringen Teil zu Schwefelsäure oxydiert. Weil also nur sehr wenig Schwefelsäure gebildet wird, stimmt in meerwasserfreiem «Magensaft» die Alkalität der Asche mit dem Salzsäureverlust nahezu überein. Noch besser wird die Übereinstimmung, wenn man die geringe gebildete Schwefelsäuremenge vom Chlorverlust abzieht.

Der Chlorverlust zeigte sich aber bei diesem Versuch nicht größer als in Versuch J. Hier wurde nämlich in der Asche gleichfalls 0,0437 n Chlor gefunden. Durch die Zugabe der $20 \mathrm{ccm} \mathrm{NaCl} 0,1 \mathrm{n}$ hat sich also der Chlorverlust nicht vermehrt. Damit in bester Übereinstimmung ist, daß auch die Alkalität der Asche in beiden Fällen gleich war.

Jetzt wurde zum «Magensaft» Meerwasser zugesetzt.

Versuch IV. $10 \mathrm{ccm}$ Lösung $+10 \mathrm{ccm}$ Hochseewasser aus dem Golfe von Neapel. ${ }^{1}$ ) Die mit $4,1 \mathrm{ccm} \mathrm{NaOH}$ neutralisierte Lösung wurde zur Trockene eingedampft und der Trockenrückstand verascht. Die Asche enthielt $0,01145 \mathrm{~g} \mathrm{SO}_{3}$ (gefunden $0,0334 \mathrm{~g} \mathrm{BaSO}$ ). Das Meerwasser hatte einen $\mathrm{SO}_{3}$-Gehalt von $2,475 \mathrm{~g}$ pro Liter. Es waren also 0,02475 $-0,01145=0,0133 \mathrm{~g} \mathrm{SO}_{3}$ entwichen. ${ }^{2}$ )

Dieser Versuch zeigt, daß unter Umständen beim Ver- : brennen eine beträchtliche Menge Schwefelsäure aus dem Magensaft entweichen kann. Bei den Versuchen mit Magensaft von Scyllium haben wir den Schwefelsäureverlust beim Verbrennen leider meistens nicht bestimmt; wir glaubten damals den Einfluß der Schwefelsäureverflüchtigung vernachlässigen zu können. In einem Fall haben wir den Schwefelsäureverlust mit-

1) Siehe S. 299.

2) Beim Einäschern in einer offenen flachen Platinschale werden möglich gebildete Sulfide wohl entweder zu Oxyd oder zu Sulfat oxydiert. 
bestimmt. Wir kommen darauf bei der Besprechung der bezüglichen Untersuchung zurück.

Zur weiteren Beantwortung der Fragen 2 und 3 wurden darn folgende Versuche angestellt.

Versuch V. Von einem künstlichen Magensaft ohne Meerwasser wurden je $50 \mathrm{ccm}$ in zwei Meßkölbchen von $100 \mathrm{ccm}$ gebracht. $\mathrm{Zu}$ den ersten $50 \mathrm{ccm}$ wurden $50 \mathrm{ccm}$ Wasser gegeben. $\mathrm{Zu}$ den zweiten aber $20 \mathrm{ccm}$ Essigsäure (1,032 n) und $30 \mathrm{ccm}$ Wasser.

$10 \mathrm{ccm}$ der ersten Lösung in der gewöhnlichen Weise neutralisiert. Titrationsacidität $0,0284 \mathrm{n}$; Trockenrückstand $0,0747 \mathrm{~g}$, Asche 0,0169 g. Alkalität der Asche 0,0011 n. Chlorgehalt der Asche 0,0265 n. Gesamtchlorgehalt 0,0280. Ghlorverlust 0,0015 n. Aschenalkalität und Chlorverlust stimmen also sehr nahe überein. Der Wasserstoffionengehalt $\mathrm{C}_{\mathrm{H}}$ war $0,0109\left(\mathrm{p}_{\mathrm{H}}=1,961\right)$. Also ruft die Titrationsacidität $0,0284 \mathrm{n}$ (Salzsäure) bei einem Glühverlust von $0,0578 \mathrm{~g}$ pro $10 \mathrm{ccm}$ nur eine $\mathrm{C}_{\mathrm{H}}$ von 0,0109 hervor.

In Übereinstimmung mit dem kleineren Gehalt an Fibrinabbauprodukten sind der Chlorverlust und die Aschenalkalität kleiner als in den vorigen Versuchen. $10 \mathrm{ccm}$ der zweiten Lösung gaben eine Titrationsacidität von $0,2295 \mathrm{n}$. Alkalität der Asche 0,1982 $\mathrm{n}$; Chlorgehalt der Asche 0,0272 n. Gesamtchlorgehalt 0,0289. Chlorverlust 0,0017 n. Die ursprüngliche Titrationsacidität hätte $0,0284+0,2064=0,2348 \mathrm{n}$ sein sollen. Statt dessen gefunden $0,2295 \mathrm{n}$. Die Differenz $(0,0053 \mathrm{n}) \mathrm{muß}$ der Schwierigkeit des genauen Ermittelns des Titrationsendpunktes zugeschrieben werden, die Hydrolyse der essigsauren Salze macht, daß die Reaktion sich sehr allmählich der neutralen nähert, auch wird der neutrale Punkt schon erreicht, noch bevor eine mit der Essigsäure äquivalente Basenmenge zugegeben ist. Immerhin ist die Differenz nicht groß. Die Alkalität der Asche 0,1982 n, vermindert um den Chlorverlust $0,0017 \mathrm{n}$, also $0,1965 \mathrm{n}$, nähert sich sehr der zugefügten Essigsäuremenge $0,2064 \mathrm{n}$, sie differiert davon um $0,0059 \mathrm{n}$, also noch um ein Geringes mehr, als die zu wenig zugesetzte Basenmenge beträgt.

Der Chlorverlust ist, in Übereinstimmung mit der relativ großen Menge Carbonat der Asche, sehr klein; man hätte hier eigentlich gar keinen Chlorverlust erwarten müssen.

$\mathrm{C}_{\mathrm{H}}=0,0115 \mathrm{n}\left(\mathrm{p}_{\mathrm{H}}=1,939\right)$. Durch den großen Essigsäurezusatz ist doch die $\mathrm{C}_{\mathrm{H}}$ nur sehr wenig erhöht, wie auch zu erwarten war.

Die Kontrollversuche wurden dann fortgesetzt unter $\mathrm{Zu}$ gabe von Meerwasser, weil sich gezeigt hatte, daß der Magensaft von Scyllium einen großen Gehalt daran aufwies. Es könnte nun sein, daß die Komponenten des Meerwassersalzes aus irgend welchen Gründen die Methode unanwendbar machten. 
Dazu könnte man fragen, ob, falls es keine organischen Säuren sind, es doch wohl die Salzsäure ist, die dem Magensaft die saure Reaktion verleiht; es könnte auch eine andere anorganische Säure sein. Als solche kämen dann wohl nur Schwefelsäure oder Phosphorsäure in Betracht. Nun ist bekanntlich die Zusammensetzung des Meerwassersalzes sehr annähernd konstant. Die wichtigsten Bestandteile kommen in folgendem Verhältnis vor: ${ }^{1}$ )

\begin{tabular}{|c|c|c|c|c|c|}
\hline Chlor & Brom & $\mathrm{SO}_{3}$ & $\mathrm{CaO}$ & $\mathrm{MgO}$ & $\mathrm{SO}_{3} / \mathrm{Cl}$ \\
\hline & 0,3402 & 11,576 & 3,026 & 11,212 & 0,11576 \\
\hline
\end{tabular}

Das Verhältnis $\mathrm{SO}_{3} / \mathrm{Cl}$, wenn wir das Brom durch eine äquivalente Menge Chlors ersetzt denken, ist also 0,1158.

Bestimmen wir nun das Verhältnis $\mathrm{SO}_{3} / \mathrm{Cl}$ im Magensaft und rührt die Acidität von Schwefelsäure her, so wird das Verhältnis erhöht sein; ist aber Salzsäure die vom Magenwande sezernierte Säure, so wird es kleiner gefunden. Wenn Phosphorsäure abgeschieden worden ist, so wird diese Säure im Magensaft vorhanden sein. Das Meerwasser enthält bekanntlich nur äußerst kleine Spuren Phosphorsäure.

Für eine richtige Beurteilung war es erwünscht, das Verhältnis $\mathrm{SO}_{3} / \mathrm{Cl}$ des Meerwassers, in dem die Scyllii lebten, zu bestimmen, obgleich zu erwarten war, daß es von dem des Wasser's aus den offenen Ozeanen nicht merklich abweichen würde. Wir haben dazu sowohl im Aquariumwasser aus der zoologischen Station zu Neapel als im Meerwasser aus dem Golfe von Neapel die Schwefelsäure und das Chlor (+ Brom) genau bestimmt.

Für die Schwefelsäurebestimmungen wurden $50 \mathrm{ccm}$ pipettiert und zur Kontrolle gewogen. Die Bestimmungen geschahen weiter wie oben angegeben. ${ }^{2}$ ) Das Chlor wurde titrimetrisch nach Mohr bestimmt.

\section{Aquariumwasser.}

a) $50 \mathrm{ccm}\left(51,3220 \mathrm{~g}\right.$ korrigiert auf den leeren Raum) gaben $0,3599 \mathrm{~g} \mathrm{BaSO}_{4}$; $\mathrm{SO}_{3} \%$ 2,406, pro Liter 2,469.

1) Challenger-Expedition, Physics and Chemistry, Bd. 1, S. 138. $\mathrm{Im}$ Wasser aus der Nordsee haben wir früher für $\mathrm{SO}_{3} / \mathrm{Cl}$ Werte zwischen 0,1142 und 0,1162 gefunden, Jaarboek van het Rijksinstituut voor het onderzoek der zee, 1905, S. 112.

*) S. 294. 
b) $50 \mathrm{ccm}\left(51,3578 \mathrm{~g}\right.$ korrigiert auf den leeren Raum) gaben $0,3617 \mathrm{~g} \mathrm{BaSO}_{4}$; $\mathrm{SO}_{3} \%$ 2,416, pro Liter 2,480.

Chlor im Mittel 21,40 g (alles Halogen als Chlor berechnet) pro Liter. Verhältnis $\mathrm{SO}_{3} / \mathrm{Cl}$ im Mittel 0,1156.

Hochseewasser.

a) $50 . \mathrm{ccm}\left(51,3316 \mathrm{~g}\right.$ korrigiert) gaben $0,3607 \mathrm{~g} \mathrm{BaSO}_{4}$; $\mathrm{SO}_{3} \% 2,410$, pro Liter 2,474.

b) $50 \mathrm{ccm}\left(51,3190 \mathrm{~g}\right.$ korrigiert) gaben $0,3611 \mathrm{~g} \mathrm{BaSO}_{4}$; $\mathrm{SO}_{3} \%$ 2,414, pro Liter 2,477.

Chlor im Mittel 21,35 g pro Liter. Verhältnis $\mathrm{SO}_{3} / \mathrm{Cl}$ im Mittel 0,1160.

Das Verhältnis $\mathrm{SO}_{3} / \mathrm{Cl}$ ist also im Aquariumwasser und im Hochseewasser identisch.

Wir haben dann wieder eine Fibrin-Pepsin-Salzsäurelösung hergestellt und die obigen Versuche fortgesetzt. Diese Lösung hatte eine Titrationsacidität von $0,07346 \mathrm{n} .10 \mathrm{ccm}$ gaben, neutralisiert, einen Glühverlust von 0,216 g. Die Asche, $0,0432 \mathrm{~g}$, korrespondierte mit dem durch das Neutralisieren entstandenen $\mathrm{NaCl}(0,0430 \mathrm{~g})$.

Versuch VI. $45 \mathrm{ccm}$ der Fibrinlösung,

45 , Meerwasser, $\left.{ }^{1}\right)$

10 Wasser.

$10 \mathrm{ccm}$ neutralisiert, Titrationsacidität $0,02632-\mathrm{C}_{\mathrm{H}}=0,0014$, $\mathrm{p}_{\mathrm{H}}=2,85$.

$0,2946 \mathrm{~g}$ Trockenrückstand, $0,1192 \mathrm{~g}$ Glühverlust. Alkalität der Asche 0,0519 n. ${ }^{1}$ ) Gesamtchlorgehalt 0,2950 n; Chlorgehalt der Asche $0,2584 \mathrm{n}$, Chlorverlust $0,0366 \mathrm{n}$. Die Alkalität der Asche $(0,0519)$ differiert um $0,0153 \mathrm{n}$ vom Chlorverlust $(0,0366)$. Der Schwefelsäureverlust wurde leider nicht bestimmt. Dieser Verlust wird wahrscheinlich die Differenz decken.

Versuch VII. Wiederholung des vorigen Versuchs.

$0,2983 \mathrm{~g}$ Trockenrückstand, $0,1223 \mathrm{~g}$ Glühverlust. Ursprüngliche Titrationsacidität $0,02542, \mathrm{C}_{\mathrm{H}}=0,00136, \mathrm{p}_{\mathrm{H}}=2,865$. Alkalität der Asche 0,05295 n. Gesamtchlorgehalt 0,2950 n, Chlor der Asche 0,2593 n, Verlust $0,0360 \mathrm{n}$. Die Alkalität der Asche differiert um $0,0169 \mathrm{n}$ vom Chlorverlust; dieser Versuch stimmt also mit dem vorigen sehr gut überein.

Versuch VIII. $45 \mathrm{ccm}$ der Fibrinlösung,

$$
\begin{aligned}
45 & \searrow \text { Meerwasser, } \\
5 & \searrow \text { Essigsäure (1,032 n), } \\
5 & \searrow \text { Wasser. }
\end{aligned}
$$

$10 \mathrm{ccm}$ gaben $0,3317 \mathrm{~g}$ Trockenrückstand (nach Neutralisieren) und $0,1247 \mathrm{~g}$ Glühverlust. Ursprüngl. Titrationsacidität $0,07727 \mathrm{n}, \mathrm{C}_{\mathrm{H}}=0,00157$,

1) Dieses Meerwasser hatte einen Chlorgehalt pro Liter von 21,56 g. 
$\mathrm{p}_{\mathrm{H}}=2,805$. Alkalität der Asche 0,0552 n. Gesamtchlorgehalt 0,2950 n, Chlorgehalt der Asche 0,2937 n, Chlorverlust 0,0013 n. Der Chlorverlust ist also sehr klein und es ist wohl zu erwarten, daß auch der (nicht bestimmte) Schwefelsäureverlust klein gewesen ist. Unter Vernachlässigung dieser finden wir: $0,0552-0,0013=0,0539 \mathrm{n}$ organische Säure und $0,07727-0,0539=0,02337 \mathrm{n}$ anorganische Säure (zugefügte Essigsäure $0,0516 \mathrm{n}$, zurückgefunden $104,5 \%$ ).

\section{Versuch IX. $45 \mathrm{ccm}$ Fibrinlösung, 45 \ Meerwasser,$$
10 \text { Essigsäure (1,032 n), }
$$

$10 \mathrm{ccm}$ gaben, neutralisiert, $0,3720 \mathrm{~g}$ Trockenrückstand und $0,1392 \mathrm{~g}$ Glühverlust. Ursprüngliche Titrationsacidität $0,1235 \mathrm{n}, \mathrm{C}_{\mathrm{H}}=0,00182$, $\mathrm{p}_{\mathrm{H}}=2,739 \mathrm{~g}$. Alkalität der Asche 0,09934 n. Gesamtchlorgehalt 0,2950 n, Chlorgehalt der Asche 0,2950 n. Chlorverlust 0. Unter Vernachlässigung des (nicht bestimmten) Schwefelsäureverlusts also: 0,1235 - 0,09934 $=0,0242 \mathrm{n}$ anorganische und 0,09934 $\mathrm{n}$ organische Säure (zugefügte Essigsäure $0,1032 \mathrm{n}$, zurückgefunden $96,2 \%$ ).

$$
\begin{aligned}
& \text { Versuch X. } 45 \mathrm{ccm} \text { Fibrinlösung, } \\
& 45 \gg \text { Meerwasser. } \\
& 5 \text { Milchsäure (0,971 n). } \\
& 5 \text { \asser. }
\end{aligned}
$$

$10 \mathrm{ccm}$ gaben, neutralisiert, $0,3612 \mathrm{~g}$ Trockenrückstand und $0,1536 \mathrm{~g}$ Glühverlust. Ursprüngliche Titrationsacidität $0,0756 \mathrm{n}, \mathrm{C}_{\mathrm{H}}=0,00415$. $\mathrm{p}_{\mathrm{H}}=2,381$. Alkalität der Asche 0,0554 n. Gesamtchlorgehalt 0,2950 n, Chlorgehalt der Asche 0,2944 n, Chlorverlust 0,0006 n. Unter Vernachlässigung eventuell verflüchtigter Schwefelsäure also: 0,0554 - 0,0006 $=0,0548 \mathrm{n}$ organische und $0,0756-0,0548=0,0208 \mathrm{n}$ anorganische Säure. (Zugefügte Milchsäure $0,0486 \mathrm{n}$, zurückgefunden $0,0548 \mathrm{n}$ oder $112,8 \%$.)

Aus den Resultaten der mitgeteilten Versuche geht also hervor, daß sowohl beim Fehlen wie in Gegenwart von Meerwasser die Methode zugesetzte organische Säure mit genügender Genauigkeit zurückfinden läßt, und daß, wenn keine organische Säure vorhanden ist, auch keine vorgetäuscht wird. Man muß aber mit der Möglichkeit, daß sich außer Salzsäure auch Schwefelsäure verflüchtigt, rechnen.

Weiter ist zu bemerken, daß beim Magensaft die Säure, falls sie eine organische ist, jedenfalls eine starke sein muß und daß bei der Neutralisation also keine größeren Fehler zu befürchten sind.

Wir haben dann im künstlichen wasserhaltigen Magensaft 
das Verhältnis von $\mathrm{SO}_{3}$ / Cl bestimmt, um zu sehen, ob bei diesem Verfahren ein Plus an Chlor gefunden wird, das mit dem Salzsäuregehalt äquivalent ist. Dazu wurden die Reste der Lösungen der Versuche VI, VIII und XI, sowie von VII und X zusammengenommen.

a) (VI, VIII und IX) $50 \mathrm{ccm}$ mit $150 \mathrm{ccm}$ Wasser verdünnt, mit reiner Salzsäure angesäuert und die Schwefelsäure wie oben bestimmt. Gefunden $0,1632 \mathrm{~g} \mathrm{BaSO}_{4}$ oder $0,05598 \mathrm{~g} \mathrm{SO}_{3}$. Also auf $1000 \mathrm{ccm}$ $1,1196 \mathrm{~g} \mathrm{SO}_{\mathrm{s}}$. Hierzu gehört $1,1196 \times \frac{1}{0,1158} \mathrm{~g} \mathrm{Cl}$ oder $9,668 \mathrm{~g}$ pro Liter oder $0,2727 \mathrm{n}$. Gefunden war ein Chlorgehalt von $0,2950 \mathrm{n}$ oder $0,0223 \mathrm{n}$ mehr. Das stimmt mit dem Salzsäuregehalt (siehe Versuche VI, VII und VIII), genügend überein. ${ }^{1}$ )

b) (VII und X). Gefunden $0,1646 \mathrm{~g} \mathrm{BaSO}_{4}=0,05645 \mathrm{~g} \mathrm{SO}_{3}$. Pro Liter $1,129 \mathrm{~g} \mathrm{SO}_{s}$ und $9,730 \mathrm{~g}$ Chlor oder $0,2744 \mathrm{n}$. Gefunden $0,295 \mathrm{n}$ oder $0,0206 \mathrm{n}$ mehr.

Man konnte also erwarten, daß auch im Magensaft in dieser Weise ein Salzsäuregehalt annähernd aufgefunden werden könnte. Eine einigermaßen in Betracht kommende Resorption oder Sekretion von Chlorid oder Sulfat war im Magen nicht zu erwarten.

Durch die Vorversuche hatten sich also die Voraussetzungen als richtig erwiesen. Wir schreiten jetzt zur Beschreibung der Untersuchung der verschiedenen Magensaftproben.

Magensaft I. Zwei Scyllien wurde täglich Fibrin mit etwa $20 \mathrm{ccm}$ Meerwasser mit der Magensonde eingeführt, nach 24 Stunden wurde der Mageninhalt mit der Sonde entfernt.

Der filtrierte Saft hatte eine $C_{H}$ von $0,020, p_{H}=1,692$.

Titrationsacidität $0,0535 \mathrm{n}$. Gesamtchlor $0,5790 \mathrm{n}$. Von diesem Magensaft wurden $45 \mathrm{ccm}$ in einem kleinen Dialysator aus Pergamentpapier (Schleicher und Schüll) gegen $60 \mathrm{ccm}$ Salzsäure $(0,01985 \mathrm{n})$ dialysiert. Diese Dialyse wurde ursprünglich als ein Kompensationsdialyseversuch vorgenommen, obgleich sich bald herausstellte, daß aus mehreren Gründen die Kompensationsdialyse hier nicht zum Ziel führt, besonders wegen des hohen Meerwassergehalts.

Nach Ablauf der Dialyse gab die Untersuchung des Dialysatorinhalts : $\mathrm{C}_{\mathrm{H}}=0,0157, \mathrm{p}_{\mathrm{H}}=1,804$. Titrationsacidität $0,03248 \mathrm{n}$.

$10 \mathrm{ccm}$ gaben einen Trockenrückstand von $0,2078 \mathrm{~g}$, einen Glührückstand von $0,1548 \mathrm{~g}$. Glühverlust $0,0530 \mathrm{~g}$. Alkalität der Asche

1) Mit $\mathrm{NH}_{4} \mathrm{Cl}$ und Magnesiamixtur bleibt die Lösung ganz klar. Nach Zusatz einer Spur Phosphatlösung sofort Trübung. 
0,0405 n. Gesamtchlorgehalt 0,2517 n, Chlorgehalt der Asche 0,2191 n, Chlorverlust $0,0326 \mathrm{n}$. Die Alkalität der Asche ist um $0,0079 \mathrm{n}$ größer als der Chlorverlust.

Die den Dialysator umspülende Flüssigkeit gab folgendes :

$\mathrm{C}_{\mathrm{H}}=0,0171, \mathrm{p}_{\mathrm{H}}=1,766$. Titrationsacidität $0,03024 \mathrm{n}$.

$10 \mathrm{ccm}$ gaben einen Trockenrückstand von $0,2046 \mathrm{~g}$, einen Glührückstand von $0,1534 \mathrm{~g}$, also einen Glühverlust von $0,0512 \mathrm{~g}$. Alkalität der Asche 0,03596 n. Gesamtchlorgehalt 0,250 n. Chlorgehalt der Asche $0,2218 \mathrm{n}$, Chlorverlust $0,0282 \mathrm{n}$.

Die Alkalität der Asche ist um 0,0078 n größer als der Chlorverlust. Die Ursachen für diese Differenzen zwischen Aschenalkalität und Chlorverlust können sein: 1. der nicht in die Berechnung aufgenommene Schwefelsäureverlust; 2. ein kleiner Gehalt an organischer Säure (v. Herwerden fand früher etwas Ameisensäure); 3. Analysenfehler.

Magensaft II. Gesammelt wie Magensaft I.

$\mathrm{C}_{\mathrm{H}}=0,0212, \mathrm{p}_{\mathrm{H}}=1,674$. Titrationsacidität $0,0654 \mathrm{n}$.

$10 \mathrm{ccm}$ gaben einen Trockenrückstand von $0,4812 \mathrm{~g}$, einen Glührückstand von $0,3252 \mathrm{~g}$, also einen Glühverlust von $0,1560 \mathrm{~g}$. Alkalitäl der Asche 0,0868 n. Gesamtchlorgehalt 0,5322 n, Chlorgehalt der Asche $0,4689 \mathrm{n}$, Chlorverlust 0,0633 n.

Differenz zwischen der Alkalität der Asche und dem Chlorverlus। $0,0235 \mathrm{n}$. Sie ist hier größer infolge der größeren Konzentration des Magensaftes; Ursachen wie beim Magensaft I.

Wir haben dann zu $10 \mathrm{ccm}$ dieses Magensaftes $5 \mathrm{ccm}$ Essigsäure $(1,032 \mathrm{n})$ gegeben. Gefunden wurde :

Titrationsacidität $0,5598 \mathrm{n}^{1}$ ) $10 \mathrm{ccm}$ gaben einen Trockenrückstand von $0,8710 \mathrm{~g}$, einen Glührückstand von $0,5912 \mathrm{~g}$ und einen Glühverlust von $0,2798 \mathrm{~g}$. Gesamtchlorgehalt 0,5322 n, Chlorgehalt der Asche 0,5142 n, Chlorverlust $0,0180 \mathrm{n}$. Also, unter Vernachlässigung des Schwefelsäureverlusts, der wahrscheinlich hier sehr klein ist, $0,5280-0,0180=0,510 \mathrm{n}$ organische Säure und 0,5598 - 0,5100 =0,0498 n anorganische Säure. Die zugegebene Essigsäure ist also nahezu genau zurückgefunden (statt $0,5160 \mathrm{n}$ gefunden $0,510 \mathrm{n}$ ). Auch die anorganische Säure ist zum größten Teil aufgefunden (statt 0,0654 $\mathrm{n}$ gefunden 0,0498 n). Die Differenz ist hier noch kleiner, als ohne Essigsäurezugabe gefunden war.

Magensaft III. Gesammelt wie Magensäfte I und II, 7 Scillii, das Fibrin wurde zum Teil ohne Meerwasser eingeführt.

$\mathrm{C}_{\mathrm{H}}=0,0154, \mathrm{p}_{\mathrm{H}}=1,812$. Titrationsacidität $0,1464 \mathrm{n}$.

$10 \mathrm{ccm}$ gaben einen Trockenrückstand von $0,7758 \mathrm{~g}$, Glührückstand 0,2777 g. Glühverlust 0,4981 g. Alkalität der Asche 0,06045. Ge-

1) Bei der Berechnung wurde angenommen, daß das Anfangsvolumen statt $15 \mathrm{ccm} 10 \mathrm{ccm}$ betrug.

Die Titrationsacidität hatte eigentlich $0,5160+0,0654=0,5814 \mathrm{n}$ sein sollen. Siehe Versuch V, S. 297. 
samtchlorgehalt 0,4461 n. Chlorgehalt der Asche 0,4180 n. Chlorverlust $0,0281 \mathrm{n}$.

Differenz zwischen der Alkalität der Asche und dem Chlorverlust $0,0323 \mathrm{n}$. Ursachen dieser Differenz wie beim Magensaft I. Auf jeden Fall sieht man auch hier, daß nur sehr wenig organische Säure vorhanden sein kann.

In diesem Magensaft wurde die Sulfatschwefelsäure bestimmt. $50 \mathrm{ccm}$ wurden mit $150 \mathrm{ccm}$ Wasser verdünnt, die saure Reaktion mit etwas reiner Salzsäure verstärkt und bei Siedehitze die Schwefelsäure präzipitiert. Siehe im übrigen S. 294.

Gefunden a) $0,1778 \mathrm{~g} \mathrm{BaSO}_{4}$; b) $0,1760 \mathrm{~g} \mathrm{BaSO}_{4}$.

Mittel 0,1769 $\mathrm{g} \mathrm{BaSO}_{4}$. Also pro Liter $1,214 \mathrm{~g} \mathrm{SO}_{3}$

Im Meerwasser ist das Verhältnis $\mathrm{SO}_{3} / \mathrm{Cl}$ von uns als 0,1158 gefunden. Aus dem Schwefelsäuregehalt des Magensaftes würde man also auf einen Chlorgehalt von $\frac{1,214}{0,1158}=10,48 \mathrm{~g}$ pro Liter oder $0,2956 \mathrm{n}$ schließen. Statt dessen gefunden $0,4461 \mathrm{n}$. Also ein Plus an Chlor von $0,1505 \mathrm{n}$. Dieses Plus stimmt mit der Gesamtacidität (0,1464 n sehr nahe überein. Wir glauben darin einen weiteren Beweis $z u$ sehen für die Gegenwart von Salzsäure im Magensaft.1) Eine einigermaßen beträchtliche Resorption oder Sekretion von Sulfat oder Chlorid ist im Magen doch wohl nicht zu erwarten.

Magensaft IV. Gesammelt wie Magensaft III. Fibrin ohne Meerwasser gegeben.

$$
\mathrm{C}_{\mathrm{H}}=0,00684, \mathrm{p}_{\mathrm{H}}=2,165 \text {. Titrationsacidität } 0,1616 \mathrm{n} \text {. }
$$

$10 \mathrm{ccm}$ gaben (wie immer neutralisiert) $0,8426 \mathrm{~g}$ Trockenrückstand, $0,2650 \mathrm{~g}$ Asche, also $0,5776 \mathrm{~g}$ Glühverlust. Alkalität der Asche 0,0525 $\mathrm{n}$. Chlorgehalt der Asche 0,3940 n. Gesamtchlorgehalt im Mittel 0,4326 n. Chlorverlust $0,0386 \mathrm{n}$. Hier wurde jetzt auch der Schwefelsäureverlust der Asche bestimmt.

$10 \mathrm{ccm}-0,8506 \mathrm{~g}$ Trockenrückstand, 0,2664 g Asche. Alkalität der Asche 0,05288 n. Die Asche war dabei statt mit Schwefelsäure mit Salzsäure gelöst. Der Schwefelsäuregehalt der Asche wurde in der gewöhnlichen Weise bestimmt. Gefunden $0,0248 \mathrm{~g} \mathrm{BaSO}_{4}$. Gesamtschwefelsäure in $30 \mathrm{ccm}$ Magensaft bestimmt $0,0907 \mathrm{~g} \mathrm{BaSO}$. Also pro $10 \mathrm{ccm}$ $0,0054 \mathrm{~g} \mathrm{BaSO}_{4}$ weniger in der Asche, also Schwefelsäureverlust 0,0046 $\mathrm{n}$.

Chlor-und Schwefelsäureverlust zusammen 0,0386 +0,0046 $=0,0432 \mathrm{n}$ oder 0,0097 $\mathrm{n}$ weniger als die Aschenalkalität.

In diesem Versuch stimmt also die Alkalität der Asche bis auf etwa $0,01 \mathrm{n}$ mit den Verlusten an Salz- und Schwefelsäure. Diese kleine Differenz kann von Analysenfehlern herrühren, oder vielleicht ist auch organische Säure bis zu einem solchen geringen Betrage vorhanden.

1) Siehe auch S. 301. 
Wenn wir mit Hilfe des Verhältnisses $\mathrm{SO}_{3} / \mathrm{Cl}(=0,1158$ in Meerwasser) aus dem $\mathrm{SO}_{3}$-Gehalt wieder den Chlorgehalt des Magensaftes berechnen, so finden wir $0,2526 \mathrm{n}$. Wenn also $\mathrm{SO}_{3}$ und $\mathrm{Cl}$ im Magensafte nur vom Meerwasser herrührten, so müßte der Chlorgehalt $0,2526 \mathrm{n}$ sein. Statt dessen wurde gefunden 0,4326 $\mathrm{n}$ oder 0,180 $\mathrm{n}$ mehr, was mit der Titrationsacidität $0,1616 \mathrm{n}$ bis auf ein geringes übereinstimmt. Eine bessere Übereinstimmung konnte man hier nicht erwarten.

Fügen wir jetzt noch hinzu, daß wir mit Chlorammonium und Magnesiamixtur keine Phosphorsäure im Fibrinmagensaft auffinden konnten, während doch ein sehr kleiner Phosphorsäurezusatz sofort aufgefunden werden konnte, so ist auch die Phosphorsäure auszuschließen.

Aus den Versuchen glauben wir also mit Bestimmtheit schließen zu müssen, daß die Säure des Haifischmagensaftes Salzsäure ist.

Wir wollen zum Schluß noch zwei Versuche mit Magensaft, der nach Fütterung mit Sardinen gewonnen war, beschreiben.

Magensaft V. Sardinenmagensaft.

$\mathrm{C}_{\mathrm{H}}=0,00574, \mathrm{p}_{\mathrm{H}}=2,241$. Titrationsacidität $0,2296 \mathrm{n}$.

$10 \mathrm{ccm}$ gaben $0,8370 \mathrm{~g}$ Trockenrückstand, $0,3067 \mathrm{~g}$ Glührückstand und also $0,5303 \mathrm{~g}$ Glühverlust.

Alkalität der Asche 0,0489 n, Chlorgehalt der Asche 0,4341 n. Bcstimmung des Gesamtchlorgehalts mißlungen.

Aus diesem Versuch sehen wir, daß die Titrationsacidität sehr hoch, die $\mathrm{G}_{\mathrm{H}}$ aber sehr klein ist. Auch die Alkalität der Asche ist klein. Doch sagt dieser Versuch nicht soviel aus wie die vorigen, weil sich viel weniger überblicken läßt, was aus den Sardinen im Magen und bei der Analyse sich gebildet hat. Wir wissen z. B. nicht, bis zu welchem Grade der Phosphor der Sardinen zur Phosphorsäurebildung Veranlassung gegeben hat, obgleich bei dem großen Gehalt an kohlenstoffreichen Produkten wohl erwartet werden kann, daß bei der Veraschung der weitaus größte Teil des Phosphors sich verflüchtigt hat.

Magensaft VI. Gesammelt wie Magensaft V.

$\mathrm{C}_{\mathrm{H}}=0,00771, \mathrm{p}_{\mathrm{H}}=2,113$. Titrationsacidität $0,1837 \mathrm{n}$.

$10 \mathrm{ccm}$ gaben (wie immer nach Neutralisation) 0,8164 g Trockenrückstand, $0,34 \tilde{2} 2 \mathrm{~g}$ Asche, also $0,4712 \mathrm{~g}$ Glühverlust.

Alkalität der Asche 0,0643 n. Gesamtchlorgehalt 0,5255 n, Chlor der Asche 0,4924 n, Chlorverlust 0,0331.

Wenn wir von den beim Veraschen gebildeten anorganischen 
Säuren, wie Schwefelsäure und Phosphorsäure, sowie vom Schwefelsäureverlust absehen, so würde sich aus diesem Versuch ein Gehalt an organischen Säuren von $0,0643-0,0331=0,0312 \mathrm{n}$, und ein Gehalt von $0,1837-0,0312=0,1525 \mathrm{n}$ an anorganischen Säuren ergeben.

Auch bei diesem Magensaft war das große Säurebindungsvermögen auffallend. Wenigstens wenn wir annehmen, daß die Acidität von einer so starken Säure wie Salzsäure herrührt.

Wir haben noch folgenden Versuch ausgeführt, um diese Bindung näher zu beleuchten (S. 306).

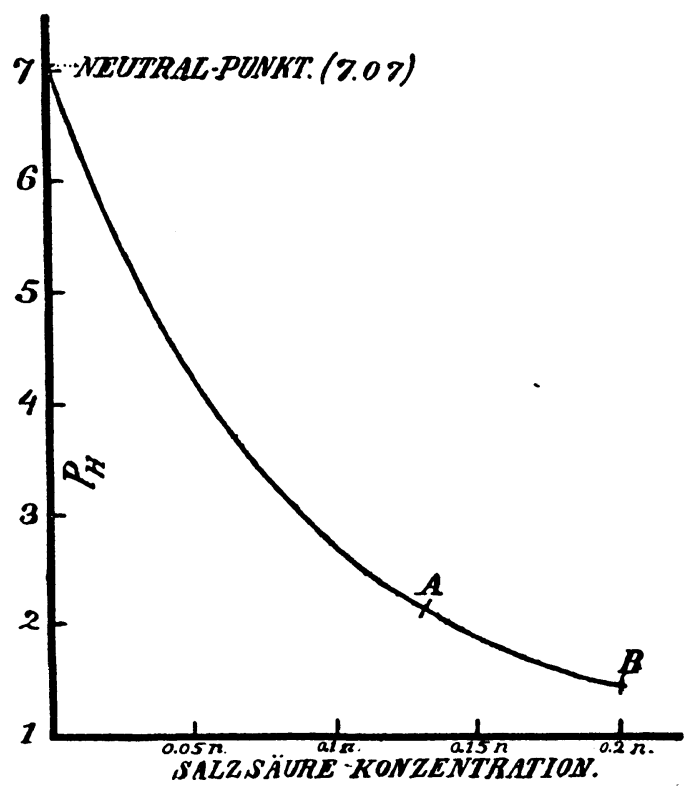

A $\ldots p_{H}$ des Magensaftes ohne Salzsäurezusatz, $6 \mathrm{ccm}$ Magensaft mit $1 \mathrm{ccm}$ Wasser verdünnt.

B ... $p_{H}$ des Magensaftes mit Salzsäurezusatz, $6 \mathrm{ccm}$ Magensaft mit $1 \mathrm{ccm}$ Salzsäure von $0,4861 \mathrm{n}$ verdünnt.

Unter der Annahme, daß die Titrationsacidität des Magensaftes bis auf ein Geringes $(0,0312 \mathrm{n})$ von Salzsäure herrührt, ist die Salzsäurekonzentration des mit Wasser verdünnten Magensaftes (A) 0,1307 $\mathrm{n}$; diejenige des mit Salzsäure verdünnten Saftes ist also 0,2001 n. Die Kurve, vom Neutralpunkt, also bei einer Salzsäurekonzentration $=0$, durch A und B gezogen, ist ganz derjenigen, die wir beim Salzsäurezusatz zu Serum erhielten, ähnlich. Wäre die ursprüngliche Säure des Magensaftes eine schwache (organische), so hätte die Kurve ein anderes Aussehen zeigen müssen.

Hoppe-Seyler's Zeitschrift f. physiol. Chemie. LXXV. 
Zu $6 \mathrm{ccm}$ des Magensaftes wurde

a) $1 \mathrm{ccm}$ Wasser,

b) 1 Salzsäure $0,4861 \mathrm{n}$ zugefügt.

In a) gefunden: $\mathrm{C}_{\mathrm{H}}=0,00698, \mathrm{p}_{\mathrm{H}}=2,156$ (ohne Verschiebung des Gleichgewichts hätte $0,00771 \times \frac{6}{7}=0,00661$ gefunden werden müssen).

In b) gefunden: $\mathrm{C}_{\mathrm{H}}=0,03428, \mathrm{p}_{\mathrm{H}}=1,465$. Die Normalität der zugefügten Salzsäure war 0,0694 n. Die der ursprünglichen Salzsäure (unter der Annahme also, daß die anorganische Säure Salzsäure ist) $0,1525 \times \frac{6}{7}=0,1307 \mathrm{n}$.

Die zugesetzte Salzsäure hat also $p_{H}$ von 2,156 auf 1,465 gebracht. Diese Änderung ist, wie die Figur (S. 305) zeigt, ganz gut mit der Annahme, daß auch die ursprüngliche Säure Salzsäure ist, in Übereinstimmung. ${ }^{1}$ )

Obgleich also die Versuche mit Sardinenmagensaft, wie oben bemerkt, etwas weniger beweisend sind, hat man doch Grund, auch hier die Salzsäure als die Säure, die zum weitaus größten Teil die Acidität bedingt, zu betrachten.

\section{Zusammenfassung.}

Es wurde betont, daß Frl. van Herwerden in ihrer ersten Abhandlung über die Magenverdauung der Fische die Unzulänglichkeit der Sjöqvistschen Methode erkannt und durch Versuche bestätigt hat.

Es wurde darauf hingewiesen, daß die Säure, die die Acidität des Magensaftes von Scyllium bedingte, keine schwache sein kann, und daß es schon deswegen unwahrscheinlich ist, daß die Acidität von einer organischen Säure herrührt.

Es wurde eine Methode angegeben, um die Frage, ob im Magensaft von Scyllium organische Säure in einigermaßen beträchtlichem Maße vorkommt, zu lösen. Eine Anzahl von Versuchen mit künstlichem Magensaft wurde ausgeführt, um

1) Man vergleiche die $P_{H}$-Kurve von Serum beim Salzsäurezusatz, Gedenkboek van van Bemmelen, S. 254 (1910). Onderzoekingen gedaan in het physiologisch Laboratorium der Utrechtsche Hoogeschool, Vijfde reeks, Bd. 11, S. 265 (1910). 
die Zuverlässigkeit der Methode z prüfen. Es hat sich dabei ergeben, daß die Methode zu rickigen Resultaten führt.

Nach dieser Methode wurder eine Anzahl von Magensaftproben untersucht. Die Untersuciung führte zu dem unzweideutigen Resultat, daß wenigsten: zum weitaus größten Teil die Acidität dieses Magensaftes va Salzsäure herrühren muß.

Dieses Resultat wurde nochauf andere Weise bestätigt, so durch den Vergleich des gesanten Chlorgehalts mit demjenigen, der sich aus dem Schweftsäuregehalt des Magensaftes berechnen läßt, unter der Annahm, daß die Salz- und Schwefelsäure nur vom Meerwasser herrühten. Weiter durch das Salzsäurebindungsvermögen einer Maensaftprobe. 